\title{
Automatic Test Case Generation with SilK Testing
}

\author{
Amandeep Kaur \\ M-Tech (C.S.E.) \\ LLRIET Moga
}

\author{
Harwinder Singh Sohal \\ Assistant Professor \\ I.T. Dept., LLRIET, Moga
}

\begin{abstract}
The research in Software Testing accumulate large amount of data. As the software technology more advanced day by day, the complexity of software is increasing. Software testing is costly and time consuming process in software development life cycle. Software developer faces many problem when create test cases because if any inaccurate or incomplete step follow during create test cases then it give application failure report to user. There are different software testing techniques and new technologies available to solve the bugs in software. Automated testing solves the bugs in few seconds as compared to manual testing. Manual testing process very slow because many logs and debug points used by user to find bugs in software. Many tools are available for automated test case generation. The main purpose of this paper how do automatic testing with silk tool. Silk testing is a new way to create test cases. Silk testing support to web applications and java based applications and traditional client server based applications. Silk testing uses any browser to execute the report of test cases. With the help of test cases it is easy to find the faults in software. The research work of this paper is when user selects objects as they navigate through the application then side by side objects records with the help of silk testing. After all steps of application are completed then press the play button. It highlights all the objects or paths you follow in the application. Silk testing is related with real world example like song record and then play.
\end{abstract}

\section{Keywords}

Silk testing, B lack box testing, depth first search

\section{INTRODUCTION}

Silk $4 \mathrm{j}$ is an automation tool for regression and functional testing It is developed by Segue Software Inc. Automated testing tool enhance the work of software developers and reduce the role of manual testing. it is the perfect solution for complex test cases. It works for different application that is based on java or web. Test automation process increase customer satisfaction by reducing more defects. Silk testing is like a black box testing because it control all click handlings that performed by users. The competition of software development between different companies is very hard. The software producing companies want to increase the software development with high quality with cost and high efficiency. For java based silk testing requires a testing framework like eclipse IDE. The silk $4 \mathrm{j}$ is as exe file that import with eclipse. The silk $4 \mathrm{j}$ testing does either manually and automatically. The automation testing gives accurate and fast report about the test cases. There are different methods that are used to get the properties of objects like size of object and how many attributes of objects and how many functions the object performed. But silk testing is different than other methods. It create automated test case as software developer click on object. Silk testing enables us to direct database access and validation and ability to test across multiple platform bowsers and for technologies. Now silk testing is also for mobile based operating systems like android and other devices. Silk4j testing has following purposes.

1. It has developed to ensure that you testing performed effectively.

\section{It covers high proportion of defects.}

\section{How silk Test works}

The components silk test host and Silk test agent help in silk testing. The graphical user interface part of an application contains objects such as menus, list, button, text view an d a mouse to initiate application functions. Silk test execute these objects and recognize these objects as they select the object. The object recognize on the base of class (Template), attributes and function that identify them. During testing silk test interact with objects to submit operation to the application automatically then determine and verify the action of an operation.

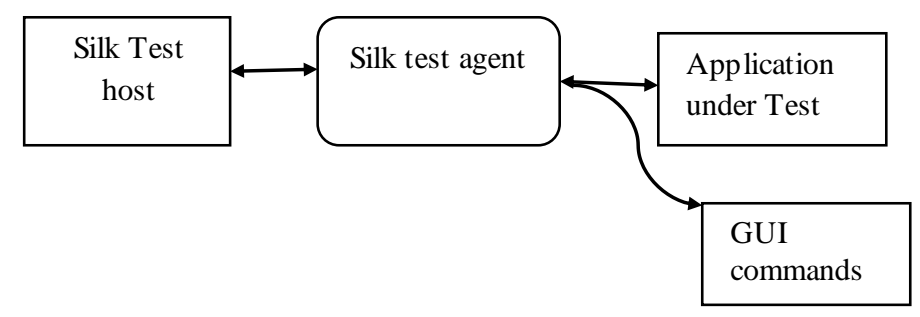

Figure 1: Process of silk testing

\section{Silk test host:}

Automation testing can be created without coding. It enables software developer to create tests in their IDE of choice. I have used eclipse ide as a silk test host. This host software is the silk test component you use to develop, edit, compile ad run, and debug your test script.

Silk test agent:

Silk test agent is also component of silk testing. It interacts with the external framework of your application like button, ListView, Spinner. When you select any specific GUI command then software test agent translates the command in your test scripts into GUI specific commands. The agent also runs on remote machine.

\section{How application work under automated test}

There are two different ways to create automated test using silk testing

1. Use the Record test case command

2. Write the test case manually

As user launches the application it explores all the steps of an application. As user navigates through the applications, the record test case command records all actions that are performed by user. It traces all the steps for verification of test cases. It handles all clicks and controls of an application. The user do easy recording of tests.

\section{RELATED WORK}

Mariusz A. Fecko and Christopher M. Lott (2002) has describes Lessons learned from automating tests for an operations support system. It explains experience gained in automating tests for an operations support system. A major portion of the effort was devoted to extending a commercial test tool so that testers could easily manipulate GUI objects on two implementations of the application. For this purpose, we developed a test automation library as support infrastructure for writing tests. The challenges and tradeoffs are discussed such as simplicity/complexity for a 
tester vs. a library developer, hiding/exposing window hierarchy to the tester, providing common methods for different types of GUI objects, transparently manipulating custom GUI widgets, and coping with data-dependent test cases. We discuss the requirements of test code reusability, maintainability, and portability, and describe the solutions we found. In addition, we offer observations about benefits and pitfalls of test automation, our recommendations for maximizing return on investment, and results from automating a variety of tests.

P.Venkata Soma Sekhar and K.Venkateswarlu (2011) derived an automation testing tool activity for software and methodologies. It describes Software producing companies want to increase their quality and efficiency. They often look at automated test tools as a part of a solution. Not many company's use test tools, the time it takes to evaluate which test tool that suites the company best costs much in both money and time. An automated test takes a lot of time to implement and introduce in the company To get the whole picture when to automate test we have looked at different test methodologies. Step-by Step Method, Product Life Cycle Method We have also investigated some general information about automated test like cost and when to automate. ATLM (Automated Test Life- Cycle Methodology) is a methodology that is used in order to take the correct decisions such as when to automate or not, (ATLM) is a structured methodology aim aiming toward ensuring a successful implementation. We also have some methods of automated tools. The Record/Playback Method, The functional Decomposition Method and The Key- Word Driven Method are a data-driven automated testing methodology. That allows developing automated test scripts that are more "generic". It requires only that the input and the expected results have been updated

Santo Carino, James H. Andrews (2012) describe about Creating Smart Test Cases from Brittle Recorded Tests. It statesTesting software with a GUI is difficult. Manual testing is costly and error-prone, but recorded test cases frequently "break" due to changes in the GUI. Test cases intended to test business logic must therefore be converted to a less "brittle" form to lengthen their useful lifespan. In this paper, we describe BlackHorse, an approach to doing this that converts a recorded test case to Java code that bypasses the GUI. The approach was implemented within the testing environment of Research in Motion. We describe the design of the toolset and discuss lessons learned during the course of the project.

\section{PROBLEM DEFINITION}

Silk testing is advanced technology that is used by many software organizations. The popularity of silk testing is limited before 2008. But in recent 2013 many software organization use silk testing for generate fast and accurate data. Silk testing is used for web applications, java applications and client server based applications. Silk testing is used for show objects of web pages of web applications and also in java applications. Silk testing like a black box testing it also check when user select the button for send input to server. The user check the input that sends by user in request form and then output produced by server is match with expected result or not. Object recognition is dynamic. The problem arise during silk testing is that SilkTest may not recognize some objects in a JavaScript page due to some technical reasons. And it may be necessary to make some modifications if testing should be shifted to other browser / operating system. The silk mobile testing is done with the help of device. Silk mobile provide automated functional testing of mobile devices simply by connecting the device you want to test. When device is connected and user launches the application then emulator open to test all the test case. If test cases fail it give a failure report. This is silk mobile interface on this user test the application it works correctly or not.

How to test

1. Simulators/Emulators like android devices

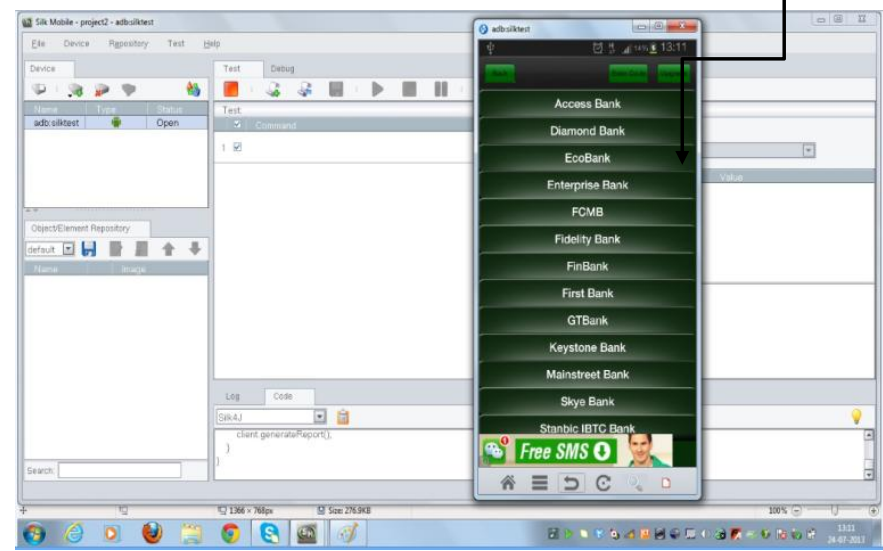

Figure 2: silk mobile testing

Now mobile device is very expensive so no anybody afford these mobile devices for testing.

2. On real devices using remote access services

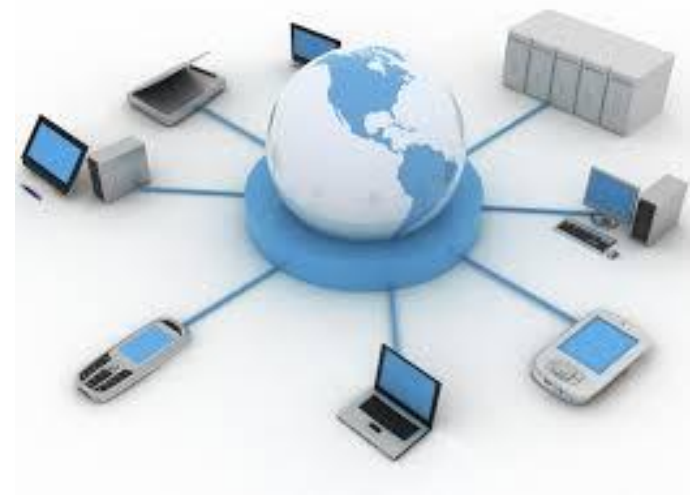

Figure 3: re mote access services

\section{OBJECTIVE}

This work has been focused to achieve the following objective

1. To study different techniques to generate automatically generate test case.

2. To study the difference between the silk mobile and silk web

3. To design the model that consumes less cost and high efficiency.

4. To design the application in java and then run on android emulator for determine the behavior of dynamic objects.

\section{PROPOSED METHODOLOGY}

How much Success gain through automation during silk mobile testing.

1. Create java based application

2. Create test cases

3. Regression and functional testing 


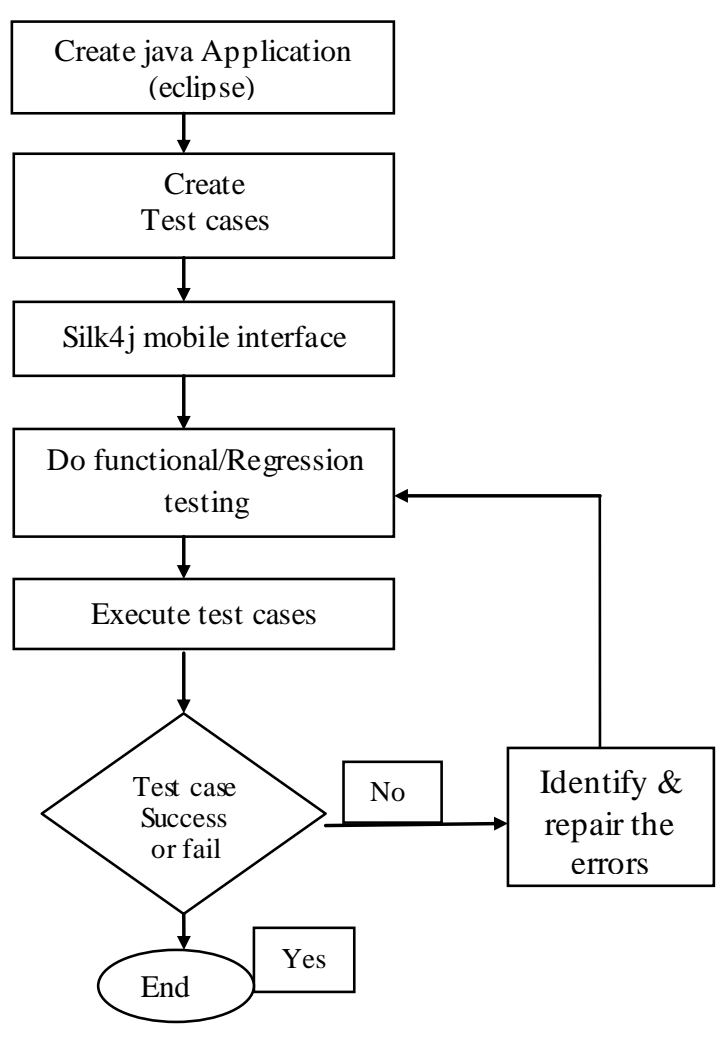

Figure 4: Flow chart of methodology

Silk testing is mostly used for functional and regression testing. Flow of silk mobile testing:

1. In SilkMobile record a test in which you perform the required interactions with the phone App which involves the entering of text.

2. The interactions will be recorded as "click on default" commands within SilkMobile. in which a click is performed on a particular element within the SilkMobile repository.

3. Select the "click on default" command which is responsible for keying of text into the search TextField - view the right hand of the screen select under "Properties".

4. Change the property from a "click element" to a "SendText" property, add a string value and save the changes (see SilkMobile Help under "Add New Operation/Command" for further details).

5. Ensure that the script replays successfully at the end of application.

6. org.silktest.mobile.test package import during silk testing.

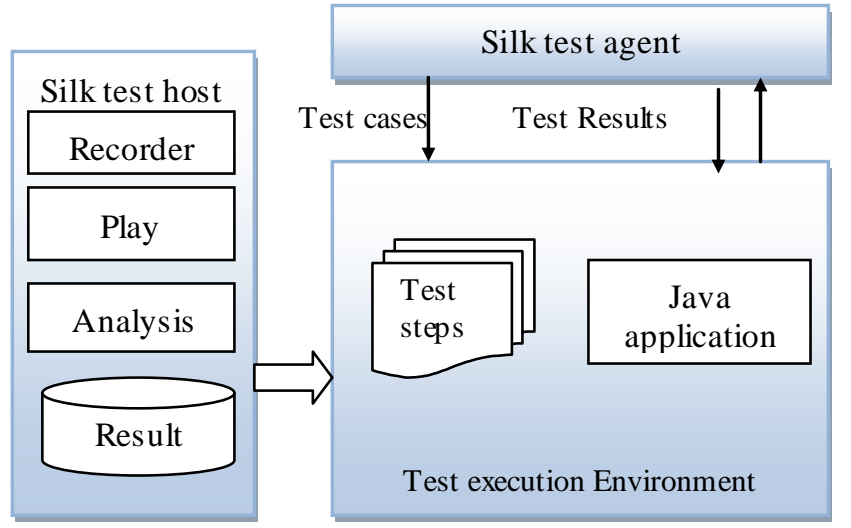

Figure 5: Silk Execution Environment

\section{CONCLUSION}

At present, java applications develop very rapidly with new technologies, but their properties, such as dynamic, multiplatform and interaction, along with the heterogeneous and autonomous running environments, make it more difficult to be maintained than the traditional software. Software testing is an effective method to ensure the quality of the java applications. But the particularities and complexities of java applications make it difficult to directly use the traditional testing theories and methods.

This paper presented our work in java application testing, which includes the requirement analy sis, test case generation and selection, testing methods and techniques,

Testing execution, and testing result.

\section{FUTURE WORK}

Future direction work on the research of testing techniques for java applications and should also pay attention on trends of java applications so improve and enhance the automatic test case generation level. Implement more new technologies for produce fast and accurate data for test case generation.

\section{REFERENCES}

[1] Mariusz A. Fecko and Christopher M. Lott (2002) "Lessons learned from automating tests for an operations support system."Applied Research Area, Telcordia Technologies, Inc., Morristown, New Jersey, USA. Pp. 1-43

[2] P.Venkata Soma Sekhar and K.Venkateswarlu (2011) "An Automated Testing Tools Activity for Software and Methodologies", International general of internet computing, pp. 54-58

[3]Santo Carino, James H. Andrews (2012) "Creating Smart Test Cases from Brittle Recorded Tests" Research in Motion, pp-89-95

Er. Amandeep Kaur received her B-Tech Degree from Punjab Technical University Jalandhar. Now doing M.Tech (CSE) from Punjab Technical University Jalandhar, India, and Research Area is Software Engineering.

\section{Er. Harwinder Singh Sohal} received his B-Tech \& M-Tech Degree from Punjab Technical University Jalandhar. $\mathrm{He}$ is working as an Assistant Professor in LLRIET Moga, Punjab. His research interests are in the fields of Congestion Control, Cloud Computing, Routing Algorithms, Routing Protocols, Load Balancing and Network Security. He has published many national and international papers. 